\title{
Effect of Eccentricity on Couette-Poiseuille Flow in Stationary and Rotating Annular Space of Different Radii Ratios
}

\author{
Reda Ameen*, Khairy Elsayed ${ }^{\dagger}$, Abdel Hamed Helali $^{\ddagger}$ and Hosny Abou-Ziyan ${ }^{\S}$
}

\begin{abstract}
This paper presents the effects of eccentricity on fluid flow in small (less than 0.3), medium (0.3-0.6) and large (0.6-0.^) radii ratios. The Couette-Poiseuille flow, in concentric and eccentric annuli with a rotating inner pipe, is investigated. Effects of eccentricity (0.0, $0.15,0.30,0.45,0.6,0.75$ and 0.90 ) on the flow structure, velocity distribution and pressure drop are investigated at radii ratios of $0.2,0.4,0.6$ and 0.8 for Reynolds number from 9780 to 288623 and Taylor number from 0 to $4.39 \times 10^{7}$. The results demonstrate that the pressure drop decreases significantly with increasing eccentricity, at radius ratio 0.8 . But, the effect of eccentricity on the pressure drop is negligible at radius ratios lower than 0.6. As wide annuli are less sensitive to the rotation of the inner cylinder than the narrow annuli, Taylor vortices were not established under the investigated parameters as a result of dominated axial velocity over tangential velocity in the considered range of parameters. The effect of eccentricity on the velocity becomes more influential as the radii ratio increases (small annuli) and vice versa. Furthermore, the effect of eccentricity is more influential on the axial and tangential velocity distributions, in the small space of the eccentric annulus. Axial velocity decreases while tangential velocity increases as the eccentricity increased.
\end{abstract}

Keywords: Couette-Poiseuille flow; radii ratio, eccentricity, pressure drop

\section{Nomenclature}

E distance between the two pipes centers

$\mathrm{P} \quad$ pressure $(\mathrm{Pa})$

$\mathrm{R} \quad$ radial distance

$\mathrm{R}_{1} \quad$ radius of inner pipe, $\mathrm{m}$

$\mathrm{R}_{2} \quad$ radius of outer pipe, $\mathrm{m}$

$\mathrm{Re} \quad$ Reynolds number based on the free-stream conditions and hydraulic diameter $\mathrm{S} \quad$ the space between the outer and inner cylinders

$\mathrm{u} \quad$ axial velocity, $\mathrm{m} / \mathrm{s}$

$\mathrm{U}_{\mathrm{b}} \quad$ bulk velocity, $\mathrm{m} / \mathrm{s}$

\footnotetext{
MSc Student, Mech. Power Eng. Dept., Faculty of Engineering, Helwan University, Cairo 11718, Egypt, redasaid826@gmail.com (corresponding author).

$\dagger$ Vrije Universiteit Brussel, Department of Mechanical Engineering, Research Group Fluid Mechanics and Thermodynamics, Pleinlaan 2, 1050 Brussels, Belgium, khairy.elsayed@ vub.ac.be; Assistant professor, Mechanical Power Engineering Department, Faculty of Engineering, Helwan University, Cairo, Egypt kelsayed75@gmail.com

* Professor, Mech. Power Eng. Dept., Faculty of Engineering, Helwan University, Cairo 11718, Egypt, abasheer00@hotmail.com

$\S \quad$ Professor, Mech. Power Eng. Dept., Faculty of Engineering, Helwan University, Cairo 11718, Egypt; Mech. Power Eng. Dept.; Faculty of Technological Studies, PAAET, Kuwait, hosnyaz@ hotmail.com
} 


$\begin{array}{ll}\mathrm{v} & \mathrm{y} \text {-velocity }(\mathrm{m} / \mathrm{s}) \\ \mathrm{W} & \text { Z-velocity }(\mathrm{m} / \mathrm{s}) \\ \mathrm{W} & \text { tangential velocity, } \mathrm{m} / \mathrm{s} \\ \mathrm{X} & \mathrm{X} \text {-coordinate } \\ \mathrm{y} & \mathrm{y} \text {-coordinate } \\ \mathrm{Z} & \mathrm{Z} \text {-coordinate } \\ \varepsilon & \text { eccentricity }=\mathrm{e} /\left(\mathrm{R}_{1}-\mathrm{R}_{2}\right) \\ \eta & \text { radius ratio }=\mathrm{R}_{1} / \mathrm{R}_{2} \\ \Delta \mathrm{p} & \text { pressure drop }(\mathrm{Pa}) \\ \mu & \text { dynamic viscosity }(\mathrm{Pa} . \mathrm{s}) \\ \rho & \text { fluid density }\left(\mathrm{kg} / \mathrm{m}^{3}\right) \\ \omega & \text { rotational speed }(\mathrm{rpm})\end{array}$

\section{Abbreviations}

CFD

DNS

LES

RANS
Computational Fluid Dynamics

Direct Numerical Simulation

Large Eddy Simulation

Reynolds Averaged Navier-Stokes

\section{Introduction}

Fluid flow in the annular space between two circular cylinders, where the inner one is rotated and the outer one is stationary, is encountered in a massive number of equipment and industrial applications. Narrow annular space exists in the clearance between stator and rotor of an electric motor or gas turbines or in the peripheral clearance of twin-screw pumps and many other applications. On the other hand, wide annular space takes place in numerous industrial applications including rotating heat exchangers, mixers and oil-well drilling (borehole and drill pipe). The radii ratios between the inner and outer circular cylinders characterize the annuli as small values are for wide annuli and larger values are for narrow annuli. Eccentricity between inner and outer cylinders creates eccentric annuli that may behave differently from concentric annuli. Therefore, understanding the flow of fluid in concentric and eccentric annuli is essential in order to predict the fluid flow and pressure drop in such annuli.

Due to the importance and wide applications of the topic, the previous work included a massive number of papers in Taylor-Couette flow and Taylor-Couette-Poiseuille flow. Work related to concentric rotating heat exchangers includes that presented by Abou-Ziyan et al. [1] for heat transfer and pressure drop in concentric annular wide channel with inner plain or finned pipe under stationary and rotating conditions in Couette-Poiseuille flow. The results proved that at a Reynolds number $\left(\operatorname{Re}=1.5 \times 10^{5}\right)$, the wide annular channel with inner pipe of helical fin spacing $75 \mathrm{~mm}$ that rotates at $400 \mathrm{rpm}$ enhances Nusselt number $(\mathrm{Nu})$ by a factor of 7.5 and also boosts the ratio of heat exchange to pumping power by a factor of 7.6, compared to the case for plain stationary pipe.

Pressure drop along with axial and tangential velocity profiles of both concentric and eccentric annulus, either stationary or rotated, received attention in literature. Adebayo and Rona [2] 
computationally investigated the force and pressure distribution in the annular space $(\eta=0.5$ and 0.44 ) between the two cylinders. The variation of the tangential velocity along the entire annulus is likely to lead to a significant axial variation in pressure distribution. Dumont et al. [3] investigated experimentally the appearance of hydrodynamic instabilities in the space between two coaxial cylinders with radius ratio (0.615) and aspect ratio (24). Moser et al. [4] investigated experimentally the combination of spatial tagging methods and a snapshot FLASH imaging sequence on the spiral flow in a concentric annuli $\left(\eta=0.0^{\circ}\right)$ with rotating inner cylinder $(\omega=10$ $\mathrm{rpm}$ ), aspect ratio (16) and Reynolds number from 0 to 11.6. Tagged images of the spiral Poiseuille flow verified that the cells in this flow propagate at a higher velocity than mean axial flow. In addition, intermittent cell formation was observed as the axial flow was increased. Rehme [5] investigated experimentally fully developed turbulent flow through three stationary concentric annuli $(\eta=0.02,0.04$ and 0.1$)$ for a Reynolds-number range from $2 \times 10^{4}$ to $2 \times 10^{5}$ ). The results showed that the position of zero shear stress is not coincident with the position of maximum velocity. Wein et al. [6] investigated numerically laminar, isothermal and axial flow in a stationary annulus and obtained velocity and viscosity profiles.

Ait-Moussa et al. [7] studied numerically flow in the annular region between rotating concentric cylinders to determine the combined effects of the co - and counter-rotation of the outer cylinder and the radius ratio on the system response. Chung and Sung [8] studied numerically, using large eddy simulation (LES), the turbulent flow in a concentric annulus with inner wall rotation at Reynolds number $=8900$. The main emphasis of this work was on the destabilization of the nearwall turbulent structures due to rotation of the inner wall. Wereley and Lueptow [9] studied experimentally the flow in the space $(\eta=0.83)$ between an inner rotating cylinder concentric with an outer stationary cylinder with an imposed pressure-driven axial flow using particle image velocimetry (PIV) in a meridional space of the annulus.

Bicalho et al. [10] investigated experimentally and numerically the pressure drop and velocity profiles through partially obstructed concentric and eccentric annuli, with and without inner tube rotation. Rotation of the inner tube results in more uniform flow distributions in the annulus, preventing flow stagnation in the smaller sector. Therefore, in the case of a partially blocked annulus with eccentricity, increasing the drill pipe rotation can improve the cuttings transport and prevent drill pipe entrapment. Hamd [11] studied numerically the effect of rotational speed, of 0 , 50, 100, 150, 200, and $250 \mathrm{rpm}$, with axial Reynolds number 200 based on bulk axial velocity with radius ratio 0.5 on the pressure gradient. Pressure gradient increases, with increasing the angular speed at eccentricity $\varepsilon=0.2$ and 0.4 . But the pressure gradient at $\varepsilon=0.2$ was greater than that of $\varepsilon=0.4$ along the considered range of rotational speed (0 to 250) rpm. Neto et al.[12] investigated numerically turbulent flows in concentric and eccentric annuli with and without rotating inner cylinder. The simulated results of axial and tangential velocities show a good agreement with the experimental data. As compared with other turbulence models, the simulations with the standard Reynolds stress model presented a slightly better prediction for most of the responses studied. Nakashima and Caetano [13] presented a review of correlations used for pressure drop calculation in narrow annular clearances with rotation of the inner cylinder. Based on these reviews, a new general correlation is proposed to calculate pressure drop through such clearances. For the non-rotating cases, the eccentricity will cause a reduction in pressure drop and an increase in axial flow. When the inner cylinder rotates, the influence of eccentricity is similar to that found in static cases if narrow channels are considered. Escudier et al. [14] studied numerically and experimentally fully developed laminar flow of a Newtonian liquid through an eccentric annulus with combined bulk axial flow and inner cylinder rotation. 
Rotation of the inner cylinder is found to have a strong influence on the axial velocity distribution, leading to two maxima in the case of a highly accented inner cylinder at high rotation speeds. Nouri and Whitelaw [15] investigated experimentally velocity components of a Newtonian and a weakly elastic shear-thinning non-Newtonian fluid in an annulus with an eccentricity of 0.5 , a radius ratio of 0.5 . The results show that the rotation had similar effects on the Newtonian and non-Newtonian fluids, with a more uniform axial flow across the annulus and the maximum tangential velocities in the narrowest space in both cases.

The survey of the published work in the open literature revealed that the effect of eccentricity on fluid flow and pressure drop in annuli of various radii ratio under fully turbulent flow conditions was not covered. Therefore, the present work addresses the influence of various eccentricities $(0.0-0.9)$ and radii ratios $(0.2-0.8)$ on fluid flow and pressure drop in both stationary and rotated inner pipe.

\section{Problem Formulation and Numerical Modeling}

The geometry of the numerical model includes annulus configuration, and exit section along with the boundary conditions, is shown in Fig. 1. The parameters of the three-dimensional model are listed in Table 1. The working fluid flowing in the annular region is air. The air viscosity $\left(\mu=1.83 \times 10^{-5}\right.$, Pa.s $)$ and density $\left(\rho=1.003, \mathrm{~kg} / \mathrm{m}^{3}\right)$. The numerical solution is carried out by solving the governing equations of mass, momentum and energy under the following assumptions; the flow is incompressible, steady and turbulent, fluid properties are constant.

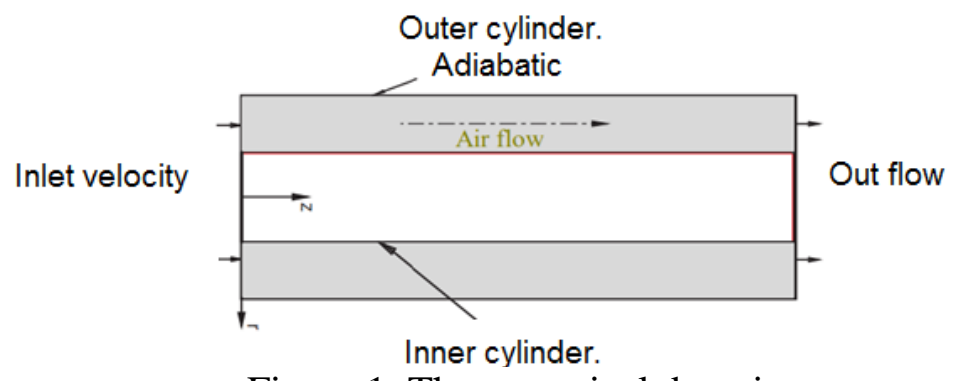

Figure 1. The numerical domain

Table 1 Model parameters

\begin{tabular}{lcc}
\hline Parameter & Symbol & Value \\
\hline Inner cylinder radius, m & $R_{1}$ & 0.025 \\
Outer cylinder radius, m & $R_{2}$ & vary \\
Cylinder length, m & $\mathrm{L}$ & 1.6 \\
Rotational speed of inner cylinder, rpm & $\mathrm{n}$ & $0-400$ \\
\hline
\end{tabular}

\section{Mathematical Modeling}

Consider an annular between two circular cylinders filled with air. The internal wall of the annular space (inner cylinder) is assumed to be at constant surface temperature, and the external wall (outer cylinder) is adiabatic. Fluid flow and heat transfer in an annulus can be described mathematically using the three dimensional governing conservations summarized as conservation of mass, conservation of momentum, and conservation of energy. The equations solved in each computational cell are Navier-Stokes equations as follows: 


\section{Conservation of mass (continuity equation):}

$$
\frac{1}{r} \frac{\partial}{\partial r}\left(r u_{r}\right)+\frac{1}{r} \frac{\partial u_{\theta}}{\partial \theta}+\frac{\partial u_{z}}{\partial z}=0
$$

Conservation of momentum equation in $\mathbf{r}, \boldsymbol{\theta}$ and, $\mathrm{z}$ directions:

$$
\begin{gathered}
\rho\left(u_{r} \frac{\partial u_{r}}{\partial r}+\frac{u_{\theta}}{r} \frac{\partial u_{r}}{\partial \theta}+u_{z} \frac{\partial u_{r}}{\partial z}-\frac{u_{\theta}^{2}}{r}\right) \\
=-\frac{\partial p}{\partial r}+\rho g_{r}+\mu\left[\frac{1}{r} \frac{\partial}{\partial r}\left(r \frac{\partial u_{r}}{\partial r}\right)+\frac{1}{r^{2}} \frac{\partial^{2} u_{r}}{\partial \theta^{2}}+\frac{\partial^{2} u_{r}}{\partial z^{2}}-\frac{u_{r}}{r^{2}}-\frac{2}{r^{2}} \frac{\partial u_{\theta}}{\partial \theta}\right] \\
\rho\left(u_{r} \frac{\partial u_{\theta}}{\partial r}+\frac{u_{\theta}}{r} \frac{\partial u_{\theta}}{\partial \theta}+u_{z} \frac{\partial u_{\theta}}{\partial z}+\frac{u_{r} u_{\theta}}{r}\right) \\
=-\frac{1}{r} \frac{\partial p}{\partial \theta}+\rho g_{\theta}+\mu\left[\frac{1}{r} \frac{\partial}{\partial r}\left(r \frac{\partial u_{\theta}}{\partial r}\right)+\frac{1}{r^{2}} \frac{\partial^{2} u_{\theta}}{\partial \theta^{2}}+\frac{\partial^{2} u_{\theta}}{\partial z^{2}}-\frac{u_{\theta}}{r^{2}}+\frac{2}{r^{2}} \frac{\partial u_{r}}{\partial \theta}\right] \\
\rho\left(u_{r} \frac{\partial u_{z}}{\partial r}+\frac{u_{\theta}}{r} \frac{\partial u_{z}}{\partial \theta}+u_{z} \frac{\partial u_{z}}{\partial z}\right)=-\frac{\partial p}{\partial \theta}+\rho g_{z}+\mu\left[\frac{1}{r} \frac{\partial}{\partial r}\left(r \frac{\partial u_{z}}{\partial r}\right)+\frac{1}{r^{2}} \frac{\partial^{2} u_{z}}{\partial \theta^{2}}+\frac{\partial^{2} u_{z}}{\partial z^{2}}\right]
\end{gathered}
$$

where $u_{r}, u_{\theta}, u_{z}$ are the velocity components in $\mathrm{r}, \theta$, and $\mathrm{z}$ directions, respectively. In addition, $p, \rho$, and $\mu$ are the fluid pressure, density, and dynamic viscosity, respectively. The governing equations (1) through (4) have been applied in the computational domain of the physical model. Turbulence is accounted for by time-averaging the above mentioned equations to produce the Reynolds Averaged Navier-Stokes (RANS) equations and to solve for the additional terms that generate using this process; a turbulence model has to be implemented.

\section{Numerical Modeling}

The present work is conducted using 3D computational fluid dynamics (CFD) model that incorporates k- $\omega$ SST turbulence model. The computational grid is illustrated in Fig. 2. A mesh sensitivity analysis is performed and a mesh size of 822,000 Cells demonstrates adequacy as listed in Table 2. In addition, the CFD model is validated against experimental results of Nouri and Whitelaw [15] and the numerical results of Neto et al. [12] as shown in Fig. 3. Where a reasonable agreement for normalized tangential (Fig. 3.a) and axial velocity (Fig. 3.b) distributions are obtained. Moreover, the computed axial velocity contours agree well with those reported by Neto et al. [12] as shown in Fig. 4.

Table 2. Predicted pressure drop, $\Delta \mathrm{P}$ for the tested numerical grids

\begin{tabular}{ccccc}
\hline No of Cells & 454250 & 584415 & 822150 & 1089375 \\
\hline$\Delta \mathrm{p}(\mathrm{Pa})$ & 18.16 & 17.93 & 17.85 & 17.84 \\
$\%$ Error in $\Delta \mathrm{p}$ & 1.79 & 0.50 & 0.06 & 0.00 \\
\hline
\end{tabular}




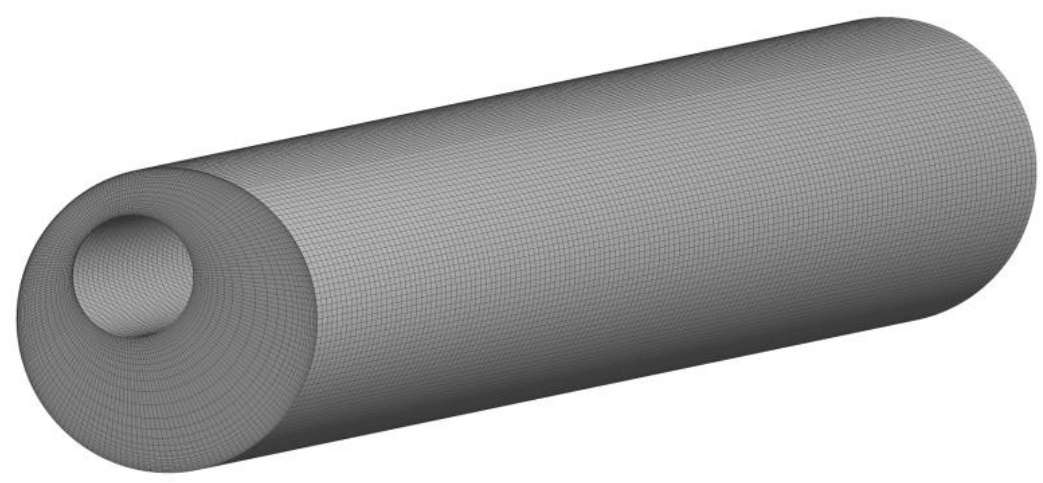

Figure 2. Eccentric computational grid

\section{$\triangle$ Nouri and Whitelaw [15] — Neto et al. [12] Present work}

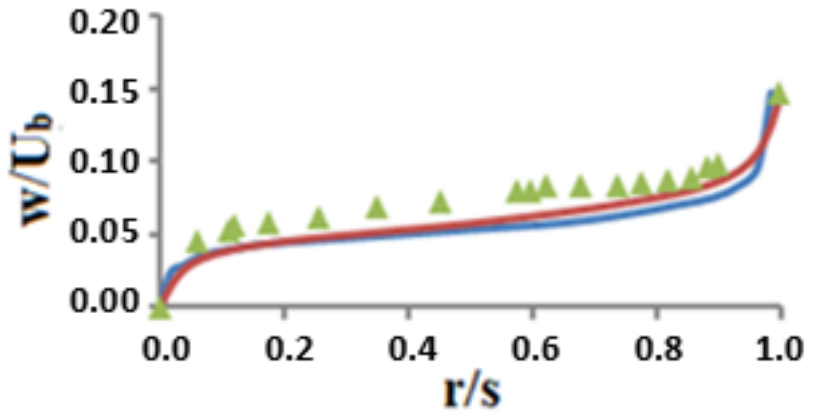

(a)

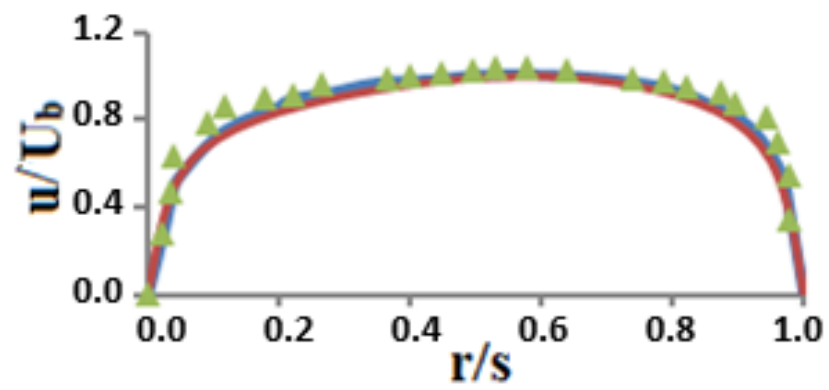

(b)

Figure 3. Validation of the numerical results

(a) normalized tangential velocity (b) normalized axial velocity

0.00 .10 .30 .40 .60 .70 .81 .01 .11 .31 .41 .51 .71 .82 .02 .12 .22 .42 .52 .72 .8

(a) Neto et al. [12]

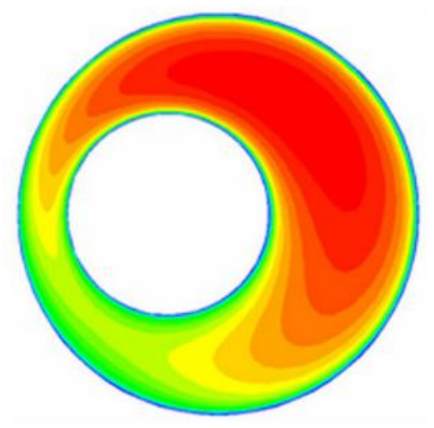

(b) Present Work

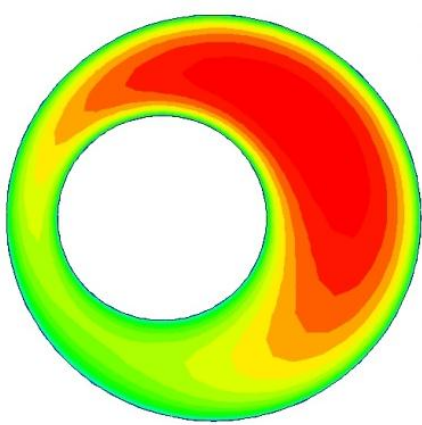

Figure 4. Axial velocity contours $(\mathrm{m} / \mathrm{s})$ at $\varepsilon=0.5$ and $\omega=300 \mathrm{rpm}$ 


\section{Results and Discussion}

\section{Flow structures}

Turbulent structures of the flow are shown in Fig. 5. The iso-values of the Q-criterion -positive second invariant of velocity gradient tensor- which is used for detecting vortex for radii ratio $\eta=$ $0.2,0.6$ and 0.8 at eccentricity $\varepsilon=0.9$ are presented in Fig. 5. The main flow is dominated by the axial Poiseuille flow, which can be attributed to the low value of the tangential velocity relative to the axial velocity. Thus, Taylor vortices do not formed in the annulus space due to small rotation effect.

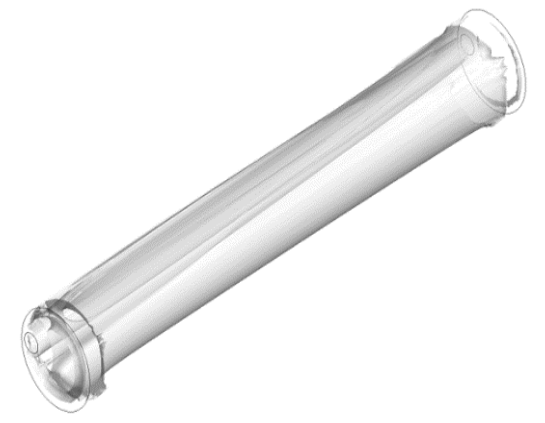

(a)

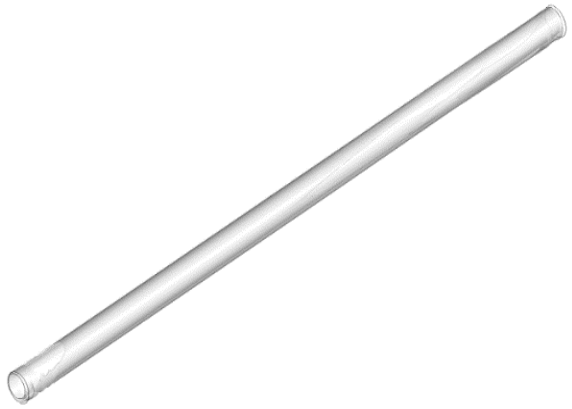

(b)

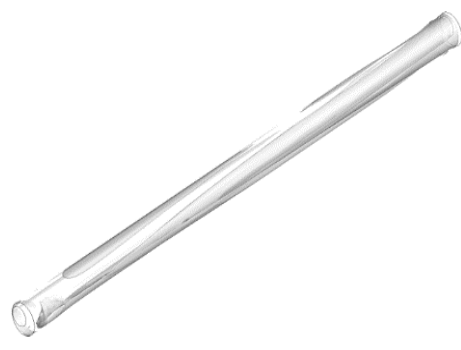

(c)

Figure 5. Iso-values of Q-criterion for various radii ratios with eccentricity $\varepsilon=0.9$

\section{Simulated Velocity Profiles}

Figure 6 shows a section of an eccentric annular space where the eccentricity splits the annulus into a largest space (P1) and a smallest space (P2). The results are taken for the spaces $\mathrm{P} 1$ and $\mathrm{P} 2$ at $\mathrm{z}=1.5 \mathrm{~m}$ for a fully developed turbulent flow (Reynolds number between 9780 and 288623) under stationary and rotating conditions (Taylor number from 0 to $4.39 \times 10^{7}$ ). The simulations were carried out for annulus with radii ratios $(\eta)$ from 0.2 to 0.8 with eccentricity $(\varepsilon)$ from 0 to $0.9\left(\varepsilon=\mathrm{e} / \mathrm{R}_{2}-\mathrm{R}_{1}\right)$, with e the distance between the centers of the inner and outer pipes and $\mathrm{R}_{1}$ and $\mathrm{R}_{2}$ the inner and outer radii, respectively.

The axial velocities are nearly in the range of $\left(1.076 \mathrm{U}_{\mathrm{b}}\right)$ to $\left(1.433 \mathrm{U}_{\mathrm{b}}\right)$ in the larger annular space (P1) for all radii ratios at all eccentricities. In the narrow space (P2) the axial velocity is reduced to approximately $\left(0.07 \mathrm{U}_{\mathrm{b}}\right)$, because the resistance to flow is increased as the space between the two pipes decreases. For the case of the directional drilling operation in well drilling, the low velocity in the narrow part causes particle settling leading to a cuttings bed formation. The same tendency was verified in Neto et al. [12].

In the case of concentric annulus, the axial and tangential velocity profiles are the same in spaces (P1 and P2) as they are equal when the eccentricity is zero. Fig. 7a shows the axial velocity and Fig. 7b shows the tangential velocity where both are normalized by the bulk velocity while the outer cylinder is stationary and the inner cylinder rotates at $200 \mathrm{rpm}$. Fig. 7a shows that the maximum normalized axial velocity has the largest value at $\eta=0.8$, medium value at $\eta=0.6$ and smallest value at $\eta=0.2$. Thus, the axial velocity profile for small radii ratio (wide annular space) shows more like a flat distribution than the profile for large radii ratio (narrow annular space) that shows a point of maximum value at $r / s=0.5$. On the other hand, Fig. $7 \mathrm{~b}$ shows that the tangential velocities at all radii ratios are equal to unity at the rotating inner cylinder $(\omega=200 \mathrm{rpm}$ and $\mathrm{r} / \mathrm{s}=1)$ and equal to zero at the outer cylinder $(\omega=0 \mathrm{rpm}$ and $\mathrm{r} / \mathrm{s}=0)$. Fig. $7 \mathrm{~b}$ shows that the 
normalized tangential velocity at $\eta=0.2$ (wide annulus) is almost zero in the annulus space up to $\mathrm{r} / \mathrm{s}$ of about 0.7 as it starts to increase and reach 1.0 at the inner cylinder radius ( $\mathrm{r} / \mathrm{s}=1)$. Thus, wide annular channels are less sensitive to the rotation of the inner cylinder than the narrow annular channels.

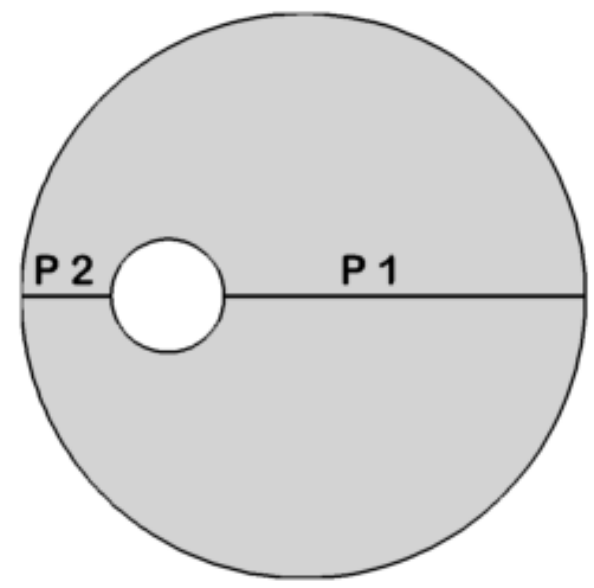

Figure 6. Eccentric annular space with spaces P1 \& P2

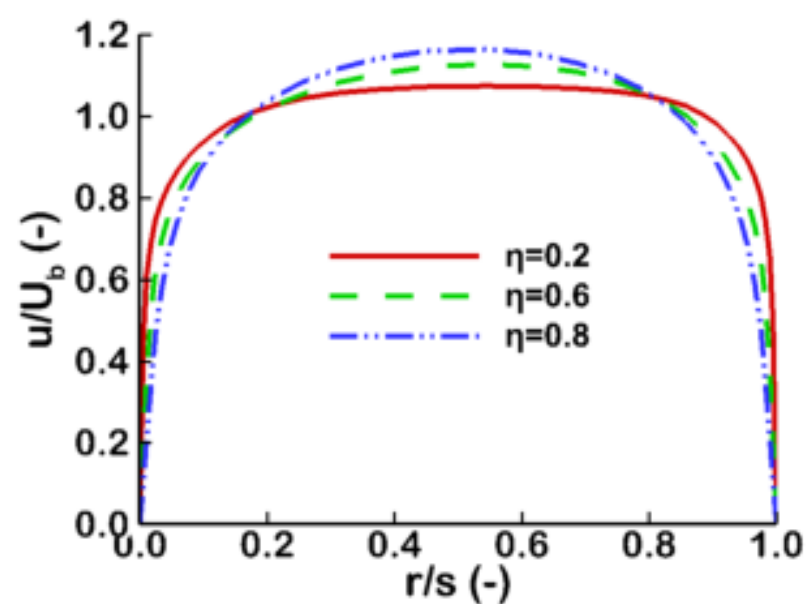

(a)

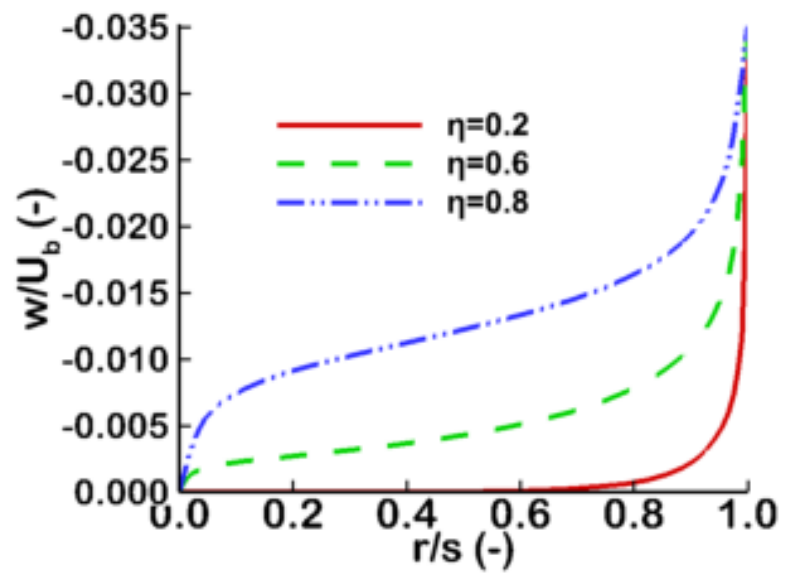

(b)

Figure 7. Simulated velocity profiles normalized by bulk velocity $\left(\mathrm{U}_{\mathrm{b}}\right)$ in concentric annulus at $\omega=200 \mathrm{rpm}$ : (a) axial velocity (b) tangential velocity

The normalized axial and tangential velocity profiles in the eccentric annulus of spaces P1 and $\mathrm{P} 2$, where $\mathrm{P} 1$ is the largest space and $\mathrm{P} 2$ is the smallest space in the annulus, for radii ratio of 0.2 , 0.6 and 0.8 are shown in Figs. 8, 9 and 10, respectively. The axial and tangential velocity at $\eta=0.2$ in the widest space (P1) are nearly the same (Figs. $8 \mathrm{a}$ and $8 \mathrm{~b}$ ) as in the concentric case (Fig. 7a and b). This indicates the negligible effect of the eccentricity on the velocity profiles for small radii ratio due to the less sensitivity of the wide space in the rotation of the inner cylinder as discussed before. On the other hand, at the smallest space (P2) the effect of eccentricity is more evident on the axial and tangential velocity distributions as presented in Figs. 8c and 8d. Clearly, as the eccentricity increases, the annulus space P2 becomes smaller. Therefore, resistance to axial flow increases and the sensitivity of the space to inner cylinder rotation increases. As a result, axial velocity decreases (Fig. 8c) while tangential velocity increases (Fig 8d) as the eccentricity increased. 
Similar trends to those discussed for $\eta=0.2$ (Fig. 8) are existing for $\eta=0.6$ (Fig. 9) and $\eta=0.8$ (Fig. 10) except that the effect of eccentricity becomes more significant at $\eta=0.6$ and 0.8 . Figs. $9 \mathrm{a}$ and $9 \mathrm{~b}$ show that the axial velocity of the wide space P1 is larger than that of the small space P2. This is due to increasing resistance to the flow in the small space. As the eccentricity increases, the space P1 increases and the space P2 decreases. Thus, while axial velocity, at $\varepsilon=0.9$, is the highest at space P1 (Fig. 9a) it becomes the lowest at space P2 (Fig. 9b). On the other hand, the tangential velocity reflects the sensitivity of the annulus to the rotation of the inner cylinder. As explained before as the annular space increases the sensitivity decreases and vice versa. Thus, as the eccentricity increases, the tangential velocity decreases in space P1 and increases in space P2 (Figs. 9c and 9d).

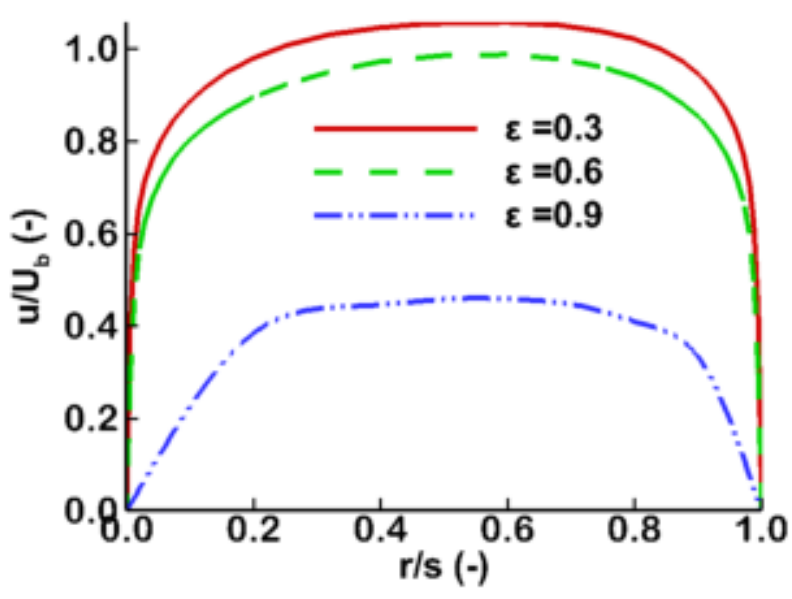

(a) Normalized axial velocity at P1

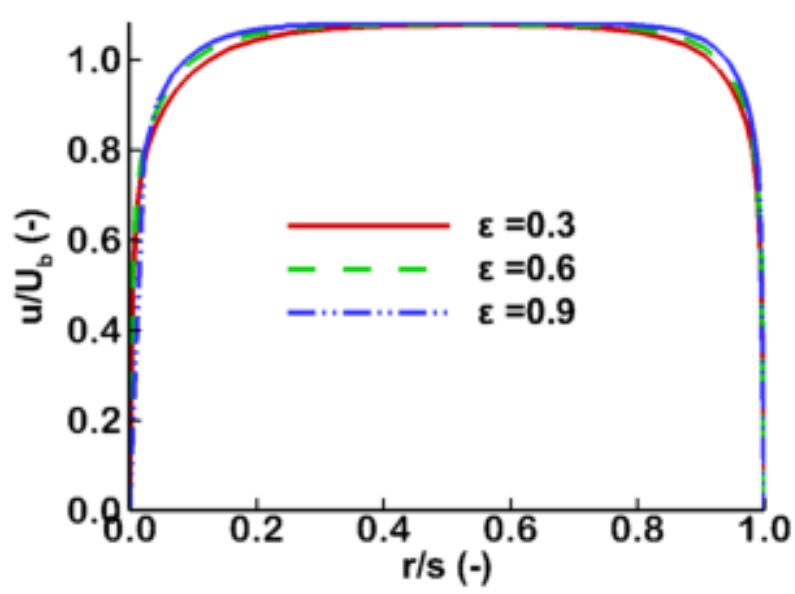

(c) Normalized axial velocity at P2

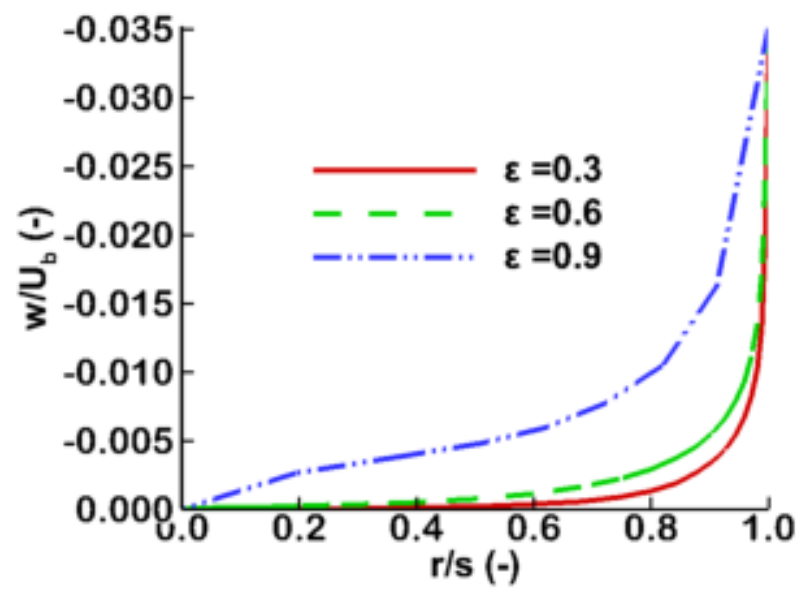

(b) Normalized tangential velocity at P1

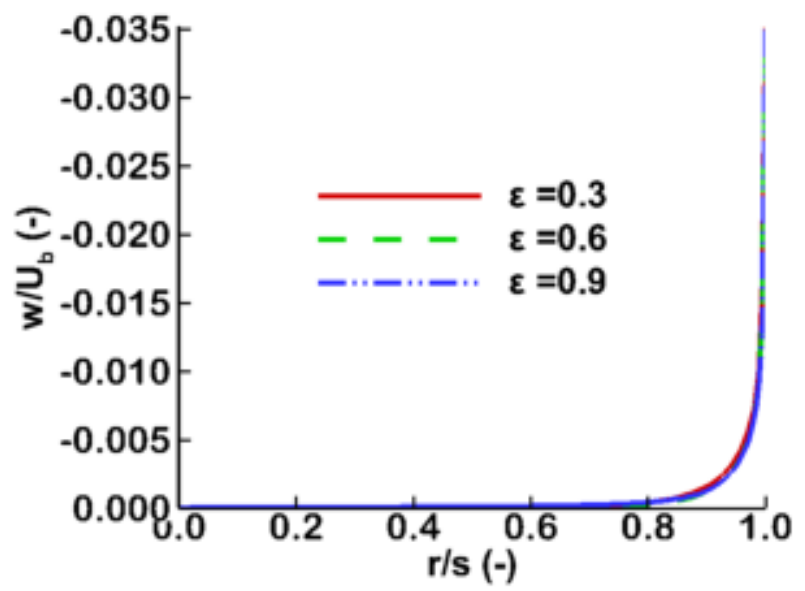

(d) Normalized tangential velocity at P2

Figure 8 . Simulated velocity profiles normalized by bulk velocity $\left(\mathrm{U}_{\mathrm{b}}\right)$ in eccentric annulus at $\eta=0.2$ and $\omega=200 \mathrm{rpm}$ : (a and b) at space P1, (c and d) at space P2 


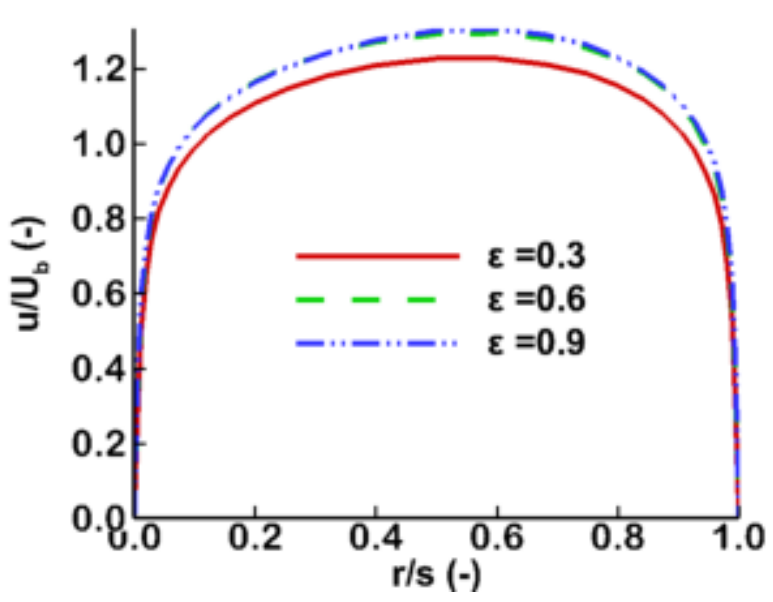

(a) Normalized axial velocity at P1

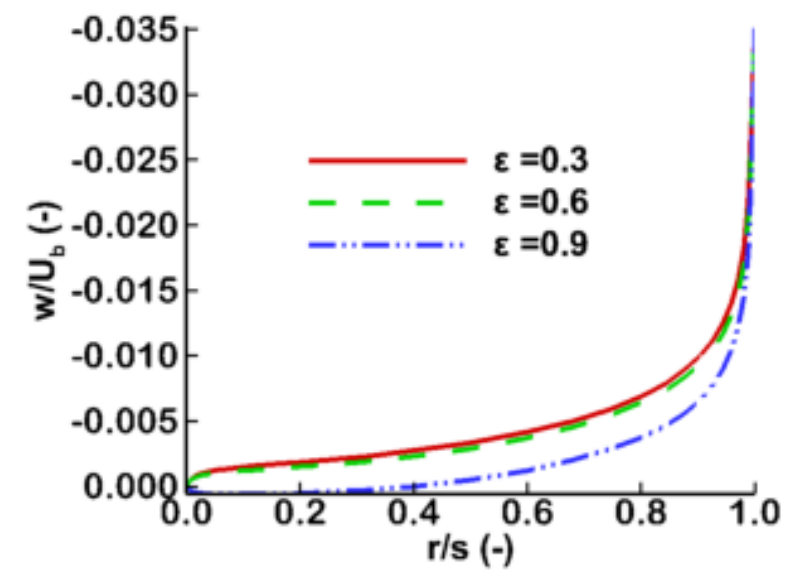

(c) Normalized tangential velocity at P1

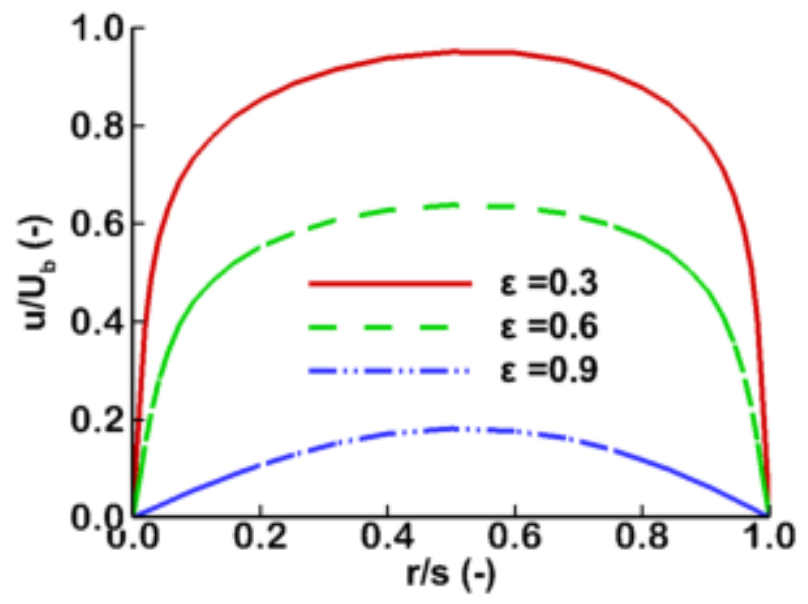

(b) Normalized axial velocity at P2

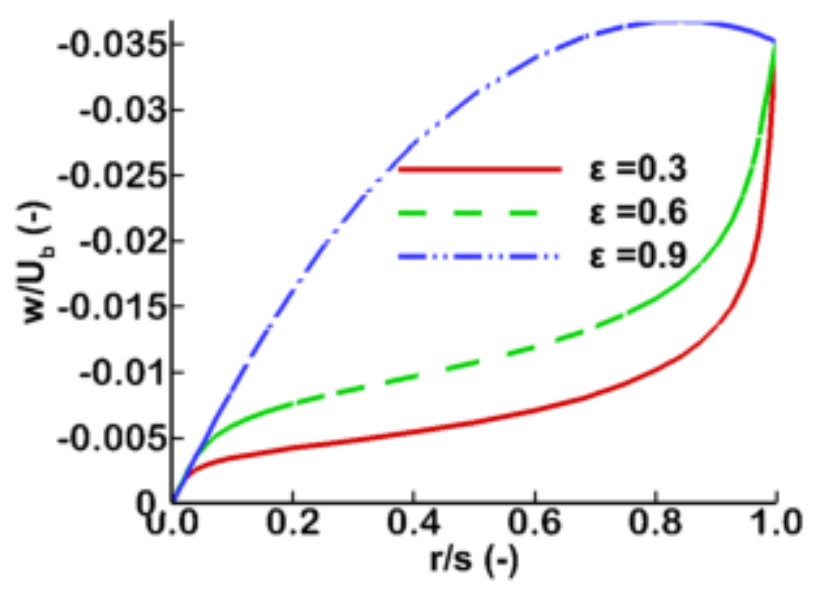

(d) Normalized tangential velocity at P2

Figure 9 Simulated velocity profiles normalized by bulk velocity $\left(\mathrm{U}_{\mathrm{b}}\right)$ in eccentric annulus at $\eta=0.6$ and $\omega=200 \mathrm{rpm}$ : (a and b) axial velocity , (c and d) tangential velocity 


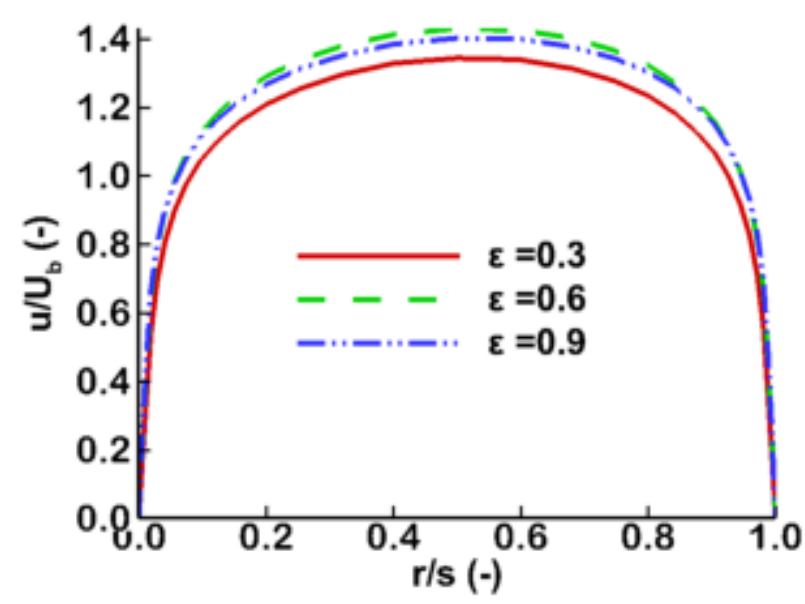

(a) Normalized axial velocity at P1

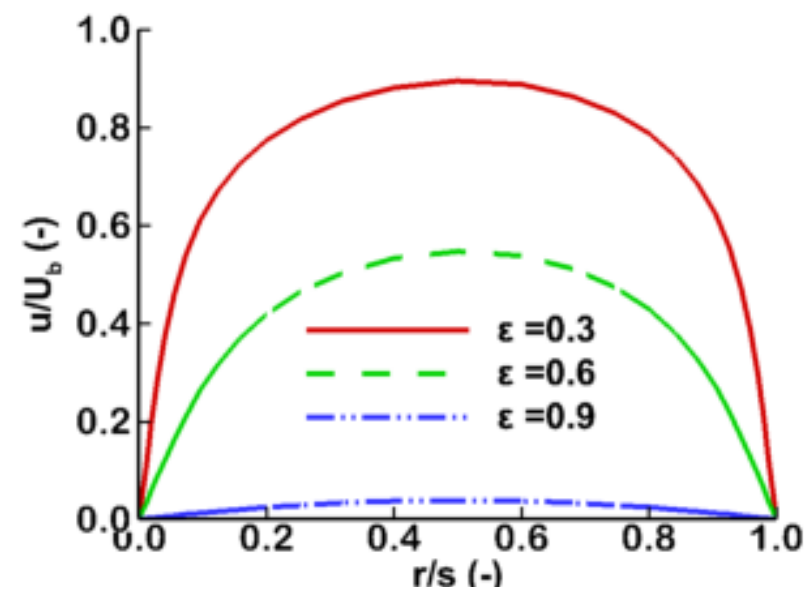

(b) Normalized axial velocity at P2

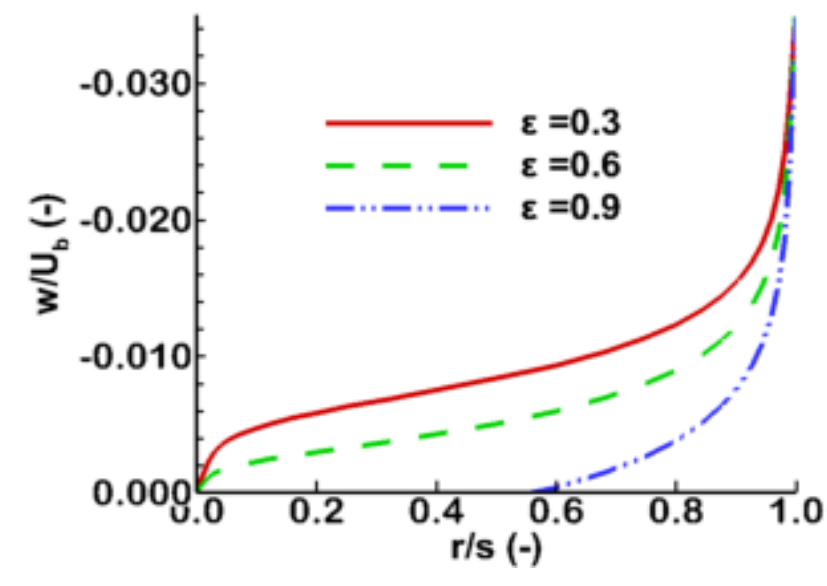

(c) Normalized tangential velocity at P1

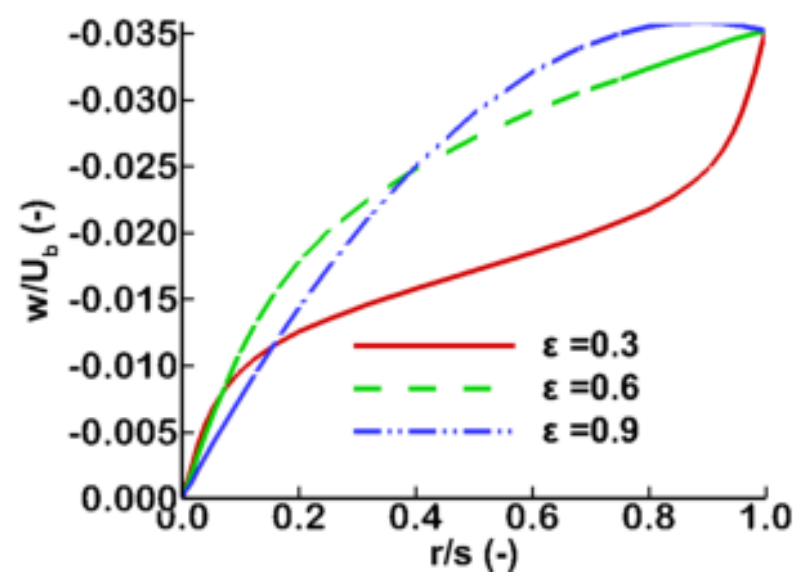

(d) Normalized tangential velocity at P2

Figure 10. Simulated velocity profiles normalized by bulk velocity $\left(\mathrm{U}_{\mathrm{b}}\right)$ in eccentric annulus at $\eta=0.8$ and $\omega=200 \mathrm{rpm}$ : (a and b) axial velocity, (c and d) tangential velocity

In addition to the normalized axial and tangential velocity distributions shown in Figs. 7-10, Figs. 11 and 12 show the axial velocity contours for $\omega=0$ and $200 \mathrm{rpm}$, respectively. These contours are taken at $\mathrm{z}=1.5 \mathrm{~m}$ when the flow becomes fully developed for radii ratios of $0.2,0.6$ and 0.8 and eccentricity of $0.0,0.3,0.6$ and 0.9 . It is indicated that the effect of eccentricity on the velocity contours becomes more influential as the radii ratio increases while the eccentricity remains constant. Also, the effect of eccentricity increases as the eccentricity ( $\varepsilon$ ) increases. However, comparing the axial velocity contours at $\omega=0 \mathrm{rpm}$ and the corresponding ones at $\omega=200 \mathrm{rpm}$ indicates similar contours in both cases. This is because the large bulk velocity $\left(\mathrm{U}_{\mathrm{b}}\right.$ $=14.87 \mathrm{~m} / \mathrm{s}$ ) dominates both cases as the tangential velocity at $\omega=200 \mathrm{rpm}$ has a small effect on the axial velocity contours. 
$\varepsilon=\mathbf{0}$
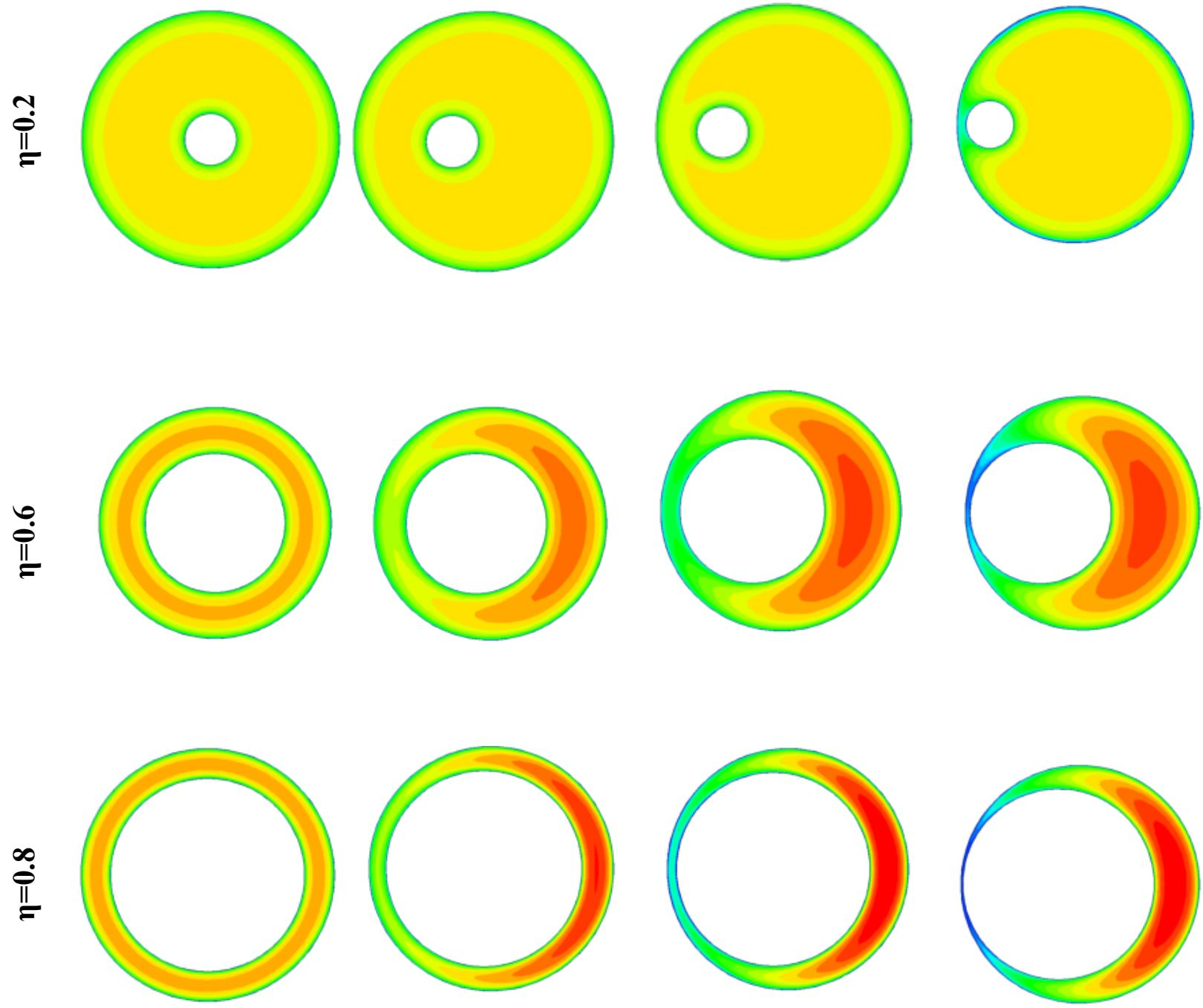

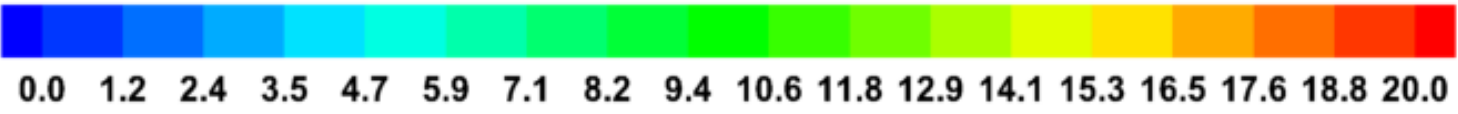

Figure 11. Axial velocity contours at $\omega=0 \mathrm{rpm}$ for eccentricities of $0.0,0.3,0.60$ and 0.90 


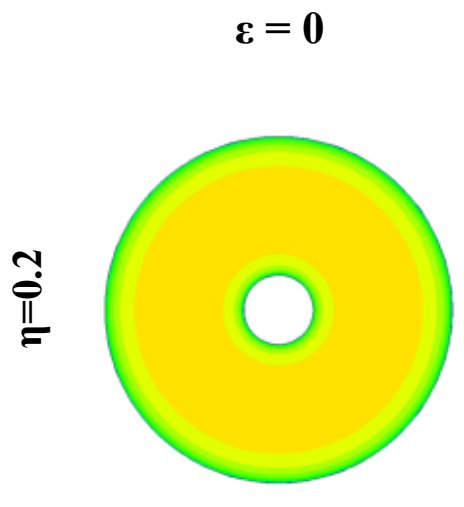

$$
\varepsilon=0.3
$$

$\varepsilon=0.6$

$\varepsilon=0.9$
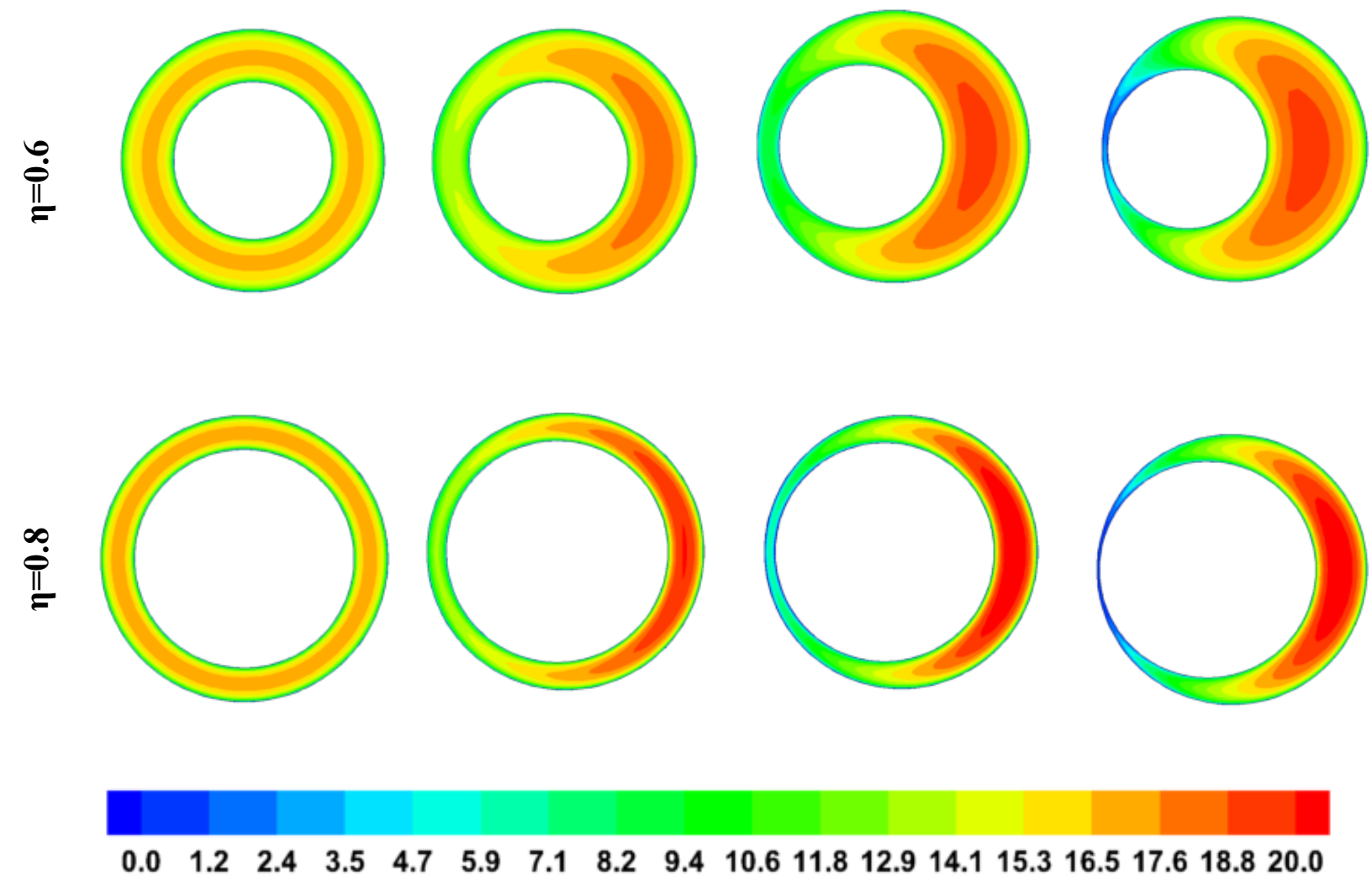

Figure 12. Axial velocity contours at $\omega=200 \mathrm{rpm}$ for eccentricities of $0.0,0.3,0.60$ and 0.90 


\section{Pressure drop}

Pressure drop is an important factor in applications which include fluid flow as it controls the pumping power required to drive the system. This in turn determines the running cost of the system. The variations of pressure drop versus eccentricity for annuli of various radii ratios rotating at 200, 300 and $400 \mathrm{rpm}$ are illustrated in Figs. 13a, 13b and 13c, respectively. The trends of pressure drop in eccentricity are similar under all rotational speeds or bulk velocities. However, the pressure drop increases substantially with increasing either the speed or the bulk velocity. It is obvious that the effect of eccentricity on the pressure drop depends on the radii ratio. It is negligible for radius ratios of 0.2 and 0.4 and mild for a radius ratio of 0.6 . But, the pressure drop is decreased significantly as the eccentricity increases at a radius ratio of 0.8 (narrow annulus space). However, the magnitude of the pressure drop increases substantially with increasing the radius ratio (narrow space) at the same eccentricity. The pressure drop at a radius ratio of 0.8 is greater than that at a radius ratio of 0.2 by more than an order of magnitude. The ratio between the pressure drops at both radii ratios is dependent on the rotational speeds and the axial velocity.

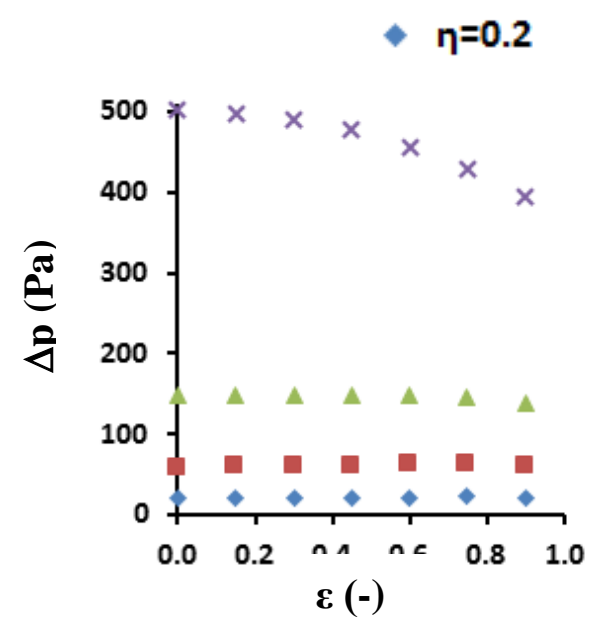

(a)

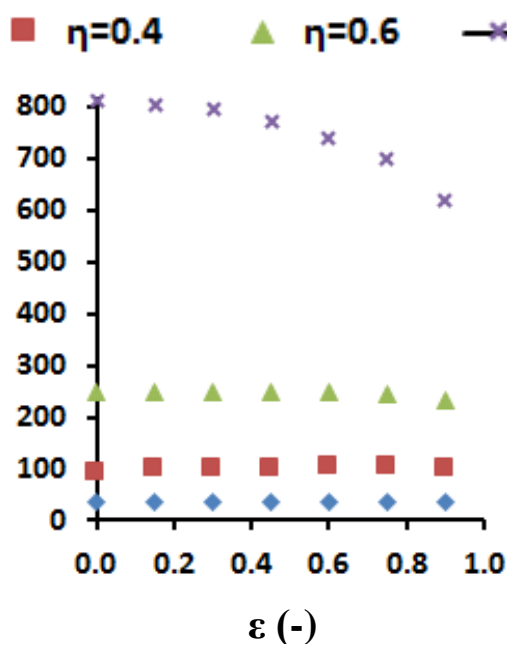

(b

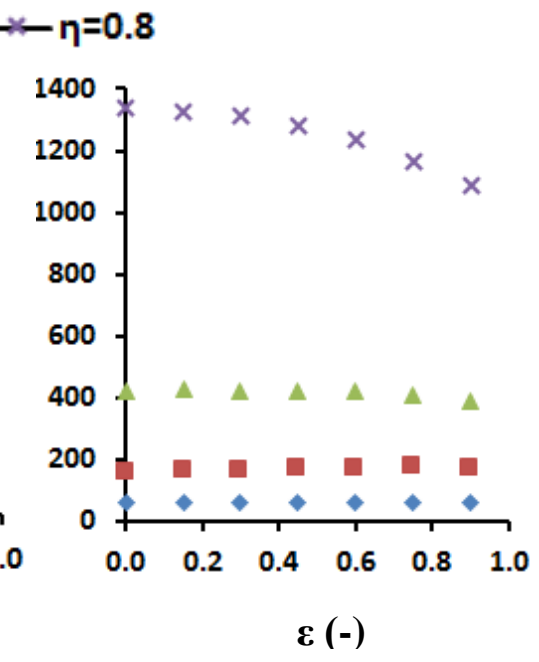

(c)

Fig. 13. Effect of $\eta$ and $\varepsilon$ on pressure drop ( $\Delta$ p): (a) $\omega=200 \mathrm{rpm} \& \mathrm{U}_{\mathrm{b}}=14.87 \mathrm{~m} / \mathrm{s}$, (b) $\omega=300 \mathrm{rpm} \& \mathrm{U}_{\mathrm{b}}=19.7 \mathrm{~m} / \mathrm{s}$, (c) $\omega=400 \mathrm{rpm} \& \mathrm{U}_{\mathrm{b}}=26.33 \mathrm{~m} / \mathrm{s}$

\section{Conclusions}

The fluid flow and pressure drop for concentric and eccentric annuli of various radii ratios are investigated for different eccentricities under stationary and rotating inner pipe using 3D CFD model. The present work is validated in terms of axial and tangential velocity profiles in an eccentric annulus $(\varepsilon=0.5)$ for a fully developed turbulent flow while the inner cylinder rotates at $\omega=300 \mathrm{rpm}$. Close agreement is established with experimental and numerical data reported in the literature. Based on the reported results, the following conclusions may be drawn:

- The main flow is dominated by the axial Poiseuille flow and Taylor vortices do not form in the annuli of radii ratios from 0.2 to 0.8 with eccentricity of 0.9 under fully developed turbulent flow when the inner cylinder rotates at 200-400 rpm. 
- The axial velocities are nearly in the range of $1.076-1.433 \mathrm{U}_{\mathrm{b}}$ in the wider annular space (P1) of the eccentric annulus for all radii ratios at all eccentricities.

- The axial velocity in the narrower space (P2) of the eccentric annulus is significantly lower $\left(0.07 \mathrm{U}_{\mathrm{b}}\right)$ than that in the wide space of the annulus.

- Wide annular channels (small radii ratio) are less sensitive to the rotation of the inner cylinder than the narrow annular channels (large radii ratio). Thus, the effect of eccentricity on the velocity becomes more influential as the radii ratio increases (small annular space) and vice versa.

- The effect of eccentricity is more influential on the axial and tangential velocity distributions, in the small space (P2) of the eccentric annulus. Axial velocity decreases while tangential velocity increases as the eccentricity increased.

- The large effect of bulk velocity $\left(\mathrm{U}_{\mathrm{b}}=14.87 \mathrm{~m} / \mathrm{s}\right)$ dominates the small effect of tangential velocity at $\omega=200 \mathrm{rpm}$. Thus, similar axial velocity contours resulted at $\omega=0$ and 200rpm.

- The pressure drop increases significantly as either the rotational speed or the bulk fluid velocity increases. The pressure drop at a radius ratio of 0.8 is greater than that at a radius ratio of 0.2 by more than an order of magnitude.

- The eccentricity effect on the pressure drop is insignificant for radius ratios of $0.2,0.4$ and 0.6. Nevertheless, the pressure drop decreases significantly as the eccentricity increases at radius ratio 0.8 .

\section{References}

[1] Abou-Ziyan, H.Z., Helali, A.H.B., and Selim, M.Y.E. "Enhancement of forced convection in wide cylindrical annular channel using rotating inner pipe with interrupted helical fins," ", International Journal of Heat and Mass Transfer, "2016, pp. 996-1007.

[2] Adebayo, D.S. and Rona, A. "The Three-Dimensional Velocity Distribution of Wide Gap Taylor-Couette Flow Modelled by CFD," ”, International Journal of Rotating Machinery, " 2016, pp. 1-11.

[3] Dumont, E., Fayolle, F., Sobolík, V., and Legrand, J. "Wall shear rate in the TaylorCouette-Poiseuille flow at low axial Reynolds number," ", International Journal of Heat and Mass Transfer, "2001, pp. 679-689.

[4] Moser, K.W., Raguin, L.G., Harris, A. et al. "Visualization of Taylor-Couette and spiral Poiseuille flows using a snapshot FLASH spatial tagging sequence," ", Magnetic Resonance Imaging, ”2000, pp. 199-207.

[5] Rehme, B.K. "Turbulent flow in smooth concentric annuli with small radius ratios,", $J$. Fluid Mech," 1974, pp. 263-287.

[6] Wein, O., Nebřenský, J., and Wichterle, K. "Non-Newtonian flow in annuli," ", Rheologica Acta," 1970, pp. 278-282.

[7] Ait-Moussa, N., Poncet, S., and Ghezal, A. "Numerical Simulations of Co- and CounterTaylor-Couette Flows: Influence of the Cavity Radius Ratio on the Appearance of Taylor 
Vortices," ", American Journal of Fluid Dynamics, ” 2015, pp. 17-22.

[8] Chung, S.Y. and Sung, H.J. "Large-eddy simulation of turbulent flow in a concentric annulus with rotation of an inner cylinder," ", International Journal of Heat and Fluid Flow, "2005, pp. 191-203.

[9] Wereley, S.T. and Lueptow, R.M. "Velocity field for Taylor - Couette flow with an axial flow," ", Physics of Fluids, " 1999, pp. 3637-3649.

[10] Bicalho, I.C., dos Santos, D.B.L., Ataíde, C.H., and Duarte, C.R. "Fluid-dynamic behavior of flow in partially obstructed concentric and eccentric annuli with orbital motion," ", Journal of Petroleum Science and Engineering, " 2016, pp. 202-213.

[11] Hamd, R.F. "Effect of Angular Velocity of Inner Cylinder on Laminar Flow through Eccentric Annular Cross Section Pipe,", , Asian Transactions on Engineering, "2013,.

[12] Neto, J.L.V., Martins, A.L., Neto, A.S., Ataíde, C.H., and Barrozo, M.A.S. "CFD applied to turbulent flows in concentric and eccentric annuli with inner shaft rotation," ", Canadian Journal of Chemical Engineering, " 2011, pp. 636-646.

[13] Nakashima, C.Y. and Caetano, E.F. "Calculation Of Pressure Drop In Narrow Rotating Annular Clearances," ", Engenharia Térmica (Thermal Engineering)," 2008, pp. 27-34.

[14] Escudier, M.P., Gouldson, I.W., Oliveira, P.J., and Pinho, F.T. "Effects of inner cylinder rotation on laminar flow of a Newtonian fluid through an eccentric annulus," ", International Journal of Heat and Fluid Flow, " 2000, pp. 92-103.

[15] Nouri, J. and Whitelaw, J. "Flow of Newtonian and Non- Newtonian Fluids in a Concentric Annuius With Rotation of the inner Cylinder," ", International Journal of Heat and Fluid Flow," 1997, pp. 236-246. 